\title{
Effect of 1 month of zopiclone on obstructive sleep apnoea severity and symptoms: a randomised controlled trial
}

\author{
Sophie G. Carter ${ }^{1}$, Jayne C. Carberry ${ }^{1}$, Garry Cho², Lauren P. Fisher ${ }^{1}$, \\ Charlotte M. Rollo', David J. Stevens², Angela L. D'Rozario ${ }^{2,3}$, \\ David K. McKenzie ${ }^{4}$, Ronald R. Grunstein ${ }^{2,5}$ and Danny J. Eckert ${ }^{1}$
}

Affiliations: ${ }^{1}$ Neuroscience Research Australia (NeuRA), Sydney, Australia. ${ }^{2}$ The Woolcock Institute of Medical Research, Sydney, Australia. ${ }^{3}$ School of Psychology, Faculty of Science, Brain and Mind Centre and Charles Perkins Centre, The University of Sydney, Sydney, Australia. ${ }^{4}$ Prince of Wales Hospital, Sydney, Australia. ${ }^{5}$ Royal Prince Alfred Hospital and Sydney Local Heath District, Camperdown, Australia.

Correspondence: Sophie G. Carter, Neuroscience Research Australia (NeuRA), PO Box 1165, Randwick, NSW 2031, Australia. E-mail: s.carter@neura.edu.au

\section{@ERSpublications}

1 month of nightly zopiclone does not worsen OSA severity or symptoms in individuals with lowmoderate arousal thresholds http://ow.ly/9JD230khJpl

Cite this article as: Carter SG, Carberry JC, Cho G, et al. Effect of 1 month of zopiclone on obstructive sleep apnoea severity and symptoms: a randomised controlled trial. Eur Respir J 2018; 52: 1800149 [https:// doi.org/10.1183/13993003.00149-2018].

ABSTRACT Hypnotic use in obstructive sleep apnoea (OSA) is contraindicated due to safety concerns. Recent studies indicate that single-night hypnotic use worsens hypoxaemia in some and reduces OSA severity in others depending on differences in pathophysiology. However, longer clinical trial data are lacking. This study aimed to determine the effects of 1 month of zopiclone on OSA severity, sleepiness and alertness in patients with low-moderate respiratory arousal thresholds without major overnight hypoxaemia.

69 participants completed a physiology screening night with an epiglottic catheter to quantify arousal threshold. 30 eligible patients (apnoea-hypopnoea index (AHI) $22 \pm 11$ events $\cdot \mathrm{h}^{-1}$ ) then completed standard in-laboratory polysomnography (baseline) and returned for two additional overnight sleep studies (nights 1 and 30$)$ after receiving either nightly zopiclone $(7.5 \mathrm{mg}$ ) or placebo during a 1-month, doubleblind, randomised, parallel trial (ANZCTR identifier ANZCTRN12613001106729).

The change in AHI from baseline to night 30 was not different between zopiclone versus placebo groups $\left(-5.9 \pm 10.2\right.$ versus $-2.4 \pm 5.5$ events $\left.\cdot \mathrm{h}^{-1} ; \mathrm{p}=0.24\right)$. Similarly, hypoxaemia, next-day sleepiness and driving simulator performance were not different.

1 month of zopiclone does not worsen OSA severity, sleepiness or alertness in selected patients without major overnight hypoxaemia. As the first study to assess the effect of a hypnotic on OSA severity and sleepiness beyond single-night studies, these findings provide important safety data and insight into OSA pathophysiology.

This article has supplementary material available from erj.ersjournals.com

This study is registered at ANZCTR with identifier number ANZCTRN12613001106729.

Received: Jan 242018 | Accepted after revision: May 232018

Copyright OERS 2018 


\section{Introduction}

Hypnotic use is common, with $3-5.5 \%$ of the general population reporting hypnotic use within the past month [1,2]. Use is higher in obese individuals (7.2\%) and the elderly (9.1\%) [1], two major risk factors for obstructive sleep apnoea (OSA), an increasingly common sleep-related breathing disorder with major adverse health and safety consequences [3-5]. Over $40 \%$ of OSA patients also have insomnia symptoms $[6,7]$.

Historically, hypnotics have been contraindicated in OSA. Major concerns have been that central nervous system depression will impair "life-saving" arousals to respiratory stimuli and worsen pharyngeal muscle contractility to promote respiratory event prolongation and hypoxaemia [8]. Indeed, certain hypnotics at high doses or in very severe, obese individuals can worsen hypoxaemia [9-11]. However, recent advances in understanding OSA pathogenesis indicate that frequent arousals destabilise sleep and breathing, and contribute to OSA $[12,13]$. Waking up too easily during airway narrowing (low respiratory arousal threshold) may be a key contributor to OSA pathogenesis in up to 50\% or more of OSA patients [12-17]. Contrary to previous beliefs, standard doses of common hypnotics (temazepam, trazodone, zopiclone and zolpidem) do not systematically reduce pharyngeal muscle contractility or increase upper airway collapsibility [11, 18, 19]. However, in two recent small physiology studies, trazodone and zopiclone did not change the apnoea-hypopnoea index (AHI), and zopiclone increased mean overnight hypoxaemia by $1 \%$ in individuals with severe OSA $[11,19]$. In contrast, eszopiclone and trazodone have been shown to promote sleep and reduce AHI without worsening event duration or hypoxaemia during single-night studies [14, 20]. Reductions in AHI were invariable in individuals with low arousal thresholds in the eszopiclone study [14]. Eszopiclone combined with oxygen also markedly reduces AHI, with greater reductions in patients with lower arousal thresholds and less anatomical compromise [21].

Given that $>50 \%$ of OSA patients fail to comply with the first-line therapy, continuous positive airway pressure $[5,17,22]$ and second-line therapies have variable and unpredictable efficacy [23], and there is increasing interest in a personalised approach to pharmacotherapy for OSA [24]. Thus, to gain important safety knowledge and understand the potential therapeutic utility of certain hypnotics for selected OSA patients, there is a need to determine if reductions in AHI are stable over time. Accordingly, the primary aim of this study was to determine if 1 month of nightly zopiclone reduces OSA severity compared with placebo in patients with low-moderate respiratory arousal thresholds and mild overnight hypoxaemia. As there is potential for residual next-day sleepiness with hypnotics, secondary aims were to assess changes in sleep parameters, daytime sleepiness and alertness to establish the safety profile of extended hypnotic use in this group of patients. Finally, to advance clinical translation, in a secondary post hoc analysis we assessed whether a recently developed clinical tool to estimate the respiratory arousal threshold from standard polysomnography (PSG) variables yielded similar findings to the gold standard, but somewhat invasive, pressure catheter approach [16].

\section{Methods}

\section{Participants}

Otherwise healthy individuals with untreated or suspected OSA aged 18-65 years were recruited via advertisement using flyers on local notice boards. Newly diagnosed patients or those who failed other therapies were also recruited from NeuRA's research volunteer database or from local sleep clinic referrals (Prince of Wales Hospital and Woolcock Sleep clinics), all located in Sydney, Australia. To be randomised, patients had to have AHI $\geqslant 5$ events. $\mathrm{h}^{-1}$, nadir arterial blood oxygen saturation $\left(\mathrm{SaO}_{2}\right) \geqslant 75 \%$ and a respiratory arousal threshold $\geqslant-25 \mathrm{cmH}_{2} \mathrm{O}$ (determined during an overnight screening physiology study, as outlined later). Other exclusion criteria included any medication that may affect breathing, sleep or pharyngeal muscle activity, pregnant or nursing mothers and known allergy to hypnotics. Participants were also asked not to consume alcohol for the duration of the 1-month trial and to allow sufficient time for sleep opportunity each night after taking the study medication.

Informed consent was provided as approved by the South Eastern Sydney Local Health District Human Research Ethics Committee. This study is registered at ANZCTR with identifier number ANZCTRN12613001106729.

\section{Protocol and key measurements}

Initially, an overnight screening physiology study (Spike 2; Cambridge Electronic Design, Cambridge, UK) with standard PSG equipment (see later) plus an epiglottic pressure catheter (Millar, Houston, TX, USA) and nasal mask was performed to quantify the arousal threshold to determine eligibility [11]. Eligible participants ( $\mathrm{AHI} \geqslant 5$ events $\mathrm{h}^{-1}$, nadir $\mathrm{SaO}_{2} \geqslant 75 \%$ and a respiratory arousal threshold $\geqslant-25 \mathrm{cmH}_{2} \mathrm{O}$ ) then completed a standard overnight PSG without an epiglottic pressure catheter. Recording equipment included: electroencephalogram, electro-oculogram, ECG, surface submentalis electromyogram, pulse 
oximeter, chest and abdominal motion, position monitor, and nasal pressure and thermistor airflow sensors (REMLogic; Natus Medical, Pleasanton, CA, USA) to re-confirm eligibility criteria and quantify baseline sleep characteristics and OSA severity in the absence of an epiglottic pressure catheter. Following the screening physiology study and baseline PSG, participants were randomised to either 1 month of nightly placebo or zopiclone $(7.5 \mathrm{mg}$ ) according to a double-blind, parallel design (figure 1). Participants returned for a standard PSG on the first (night 1) and the last (night 30) night of the 1-month trial to investigate potential changes in sleep parameters from baseline between conditions. Pill counts at night 30 were taken to assess adherence. The Epworth Sleepiness Scale (ESS), Functional Outcomes of Sleep Questionnaire (FOSQ) and Karolinska Sleepiness Scale (KSS) were administered $1.5 \mathrm{~h}$ prior to sleep at

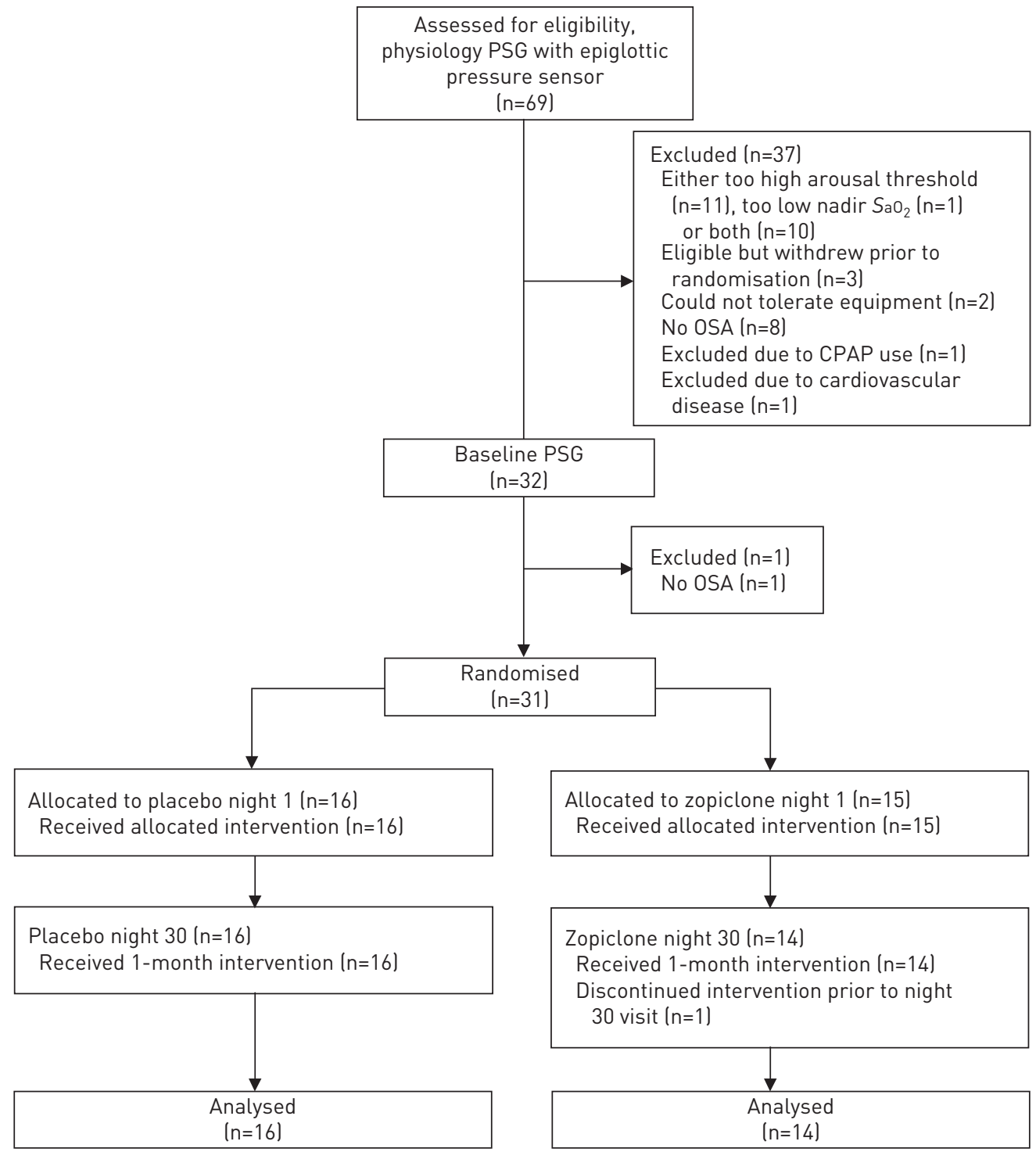

FIGURE 1 CONSORT flow diagram indicating the enrolment, randomisation, allocation, follow-up and analysis procedures for this double-blind, placebo-controlled, parallel trial. Initially, participants with a confirmed diagnosis or clinical suspicion of OSA responded to a research advertisement or were contacted via a research volunteer database. Participants attended an in-laboratory physiology screening PSG to determine eligibility for the trial. Eligible patients (untreated OSA (apnoea-hypopnoea index $\geqslant 5$ events $\cdot h^{-1}$ ), overnight nadir $\mathrm{SaO}_{2} \geqslant 75 \%$ and low-moderate respiratory arousal threshold $\left(\geqslant-25 \mathrm{cmH}_{2} \mathrm{O}\right.$ ) that were otherwise healthyl attended a standard in-laboratory baseline PSG. If still eligible following baseline, patients were then randomised to either the placebo or zopiclone condition. Participants received either once-nightly placebo or $7.5 \mathrm{mg}$ of zopiclone just prior to bed for 1 month. Participants attended two standard in-laboratory PSGs on the first (night 1) and last night (night 30) of the treatment nights. One patient was excluded from the study after the baseline PSG as they were found not to have OSA. One patient discontinued involvement in the study prior to completing night 30 due to an unrelated personal issue. PSG: polysomnography; $\mathrm{SaO}_{2}$ : arterial blood oxygen saturation; OSA: obstructive sleep apnoea; CPAP: continuous positive airway pressure. 
each visit. The KSS, Leeds Sleep Evaluation Questionnaire (Leeds) and a 30-min AusEd driving simulator task were performed each morning $30 \mathrm{~min}$ after awakening to assess next-day sleepiness and alertness [25]. At the end of the trial, participants were also asked several questions as to how they felt during the study and whether or not they believed they were in the zopiclone versus placebo arm.

\section{Data analyses}

All analyses were performed blinded to the study intervention. Sleep staging, respiratory event and arousal scoring were performed by a single experienced sleep technician according to the American Academy of Sleep Medicine recommended criteria version 2.1 [26]. Up to 20 (or as many as were available) cortical arousals that occurred in conjunction with increasing epiglottic pressure swings with obstructive apnoeas or hypopnoeas were selected at random to calculate the arousal threshold for each participant during the initial physiology screening PSG. Specifically, the nadir epiglottic pressure immediately prior to a cortical arousal was determined for each respiratory event (figure 2) and averaged to quantify the arousal threshold as described previously $[11,14]$. As an exploratory post hoc analysis, the utility of a simple validated clinical tool to estimate the arousal threshold from standard PSG variables [16] was used to determine if those with low-moderate arousal thresholds at baseline could be accurately identified.

\section{Statistical procedures}

Based on previous data (standard deviation of the difference in AHI of 6.3 events $\cdot \mathrm{h}^{-1}$ between conditions) [14], our sample size of $n=15$ in each group (zopiclone and placebo) was selected to detect a change of 7 events $h^{-1}$ from baseline to night 30 (primary outcome) between conditions with $>80 \%$ power with a two-tailed, unpaired t-test [14]. Similarly, all normally distributed (Shapiro-Wilk) data for secondary outcomes were compared using a two-tailed, unpaired t-test. Nonnormally distributed data were compared using a Wilcoxon signed-rank test. $\mathrm{p}<0.05$ was considered significant. Data are reported as mean with standard deviation or median (interquartile range) for nonnormally distributed variables.

\section{Results}

\section{Participant characteristics}

Of the 69 individuals who participated in the overnight screening physiology study, 38 were ineligible due to their arousal threshold being $\leqslant-25 \mathrm{cmH}_{2} \mathrm{O}$ and/or their nadir $\mathrm{SaO}_{2}$ being $\leqslant 75 \%$, or did not meet other inclusion criteria. Of these 38 individuals, 27 had predominantly severe OSA (AHI $48 \pm 25$ events $\cdot \mathrm{h}^{-1}$ ), nine did not have OSA (AHI $<5$ events $\cdot h^{-1}$ ) and AHI could not be quantified in two participants due to

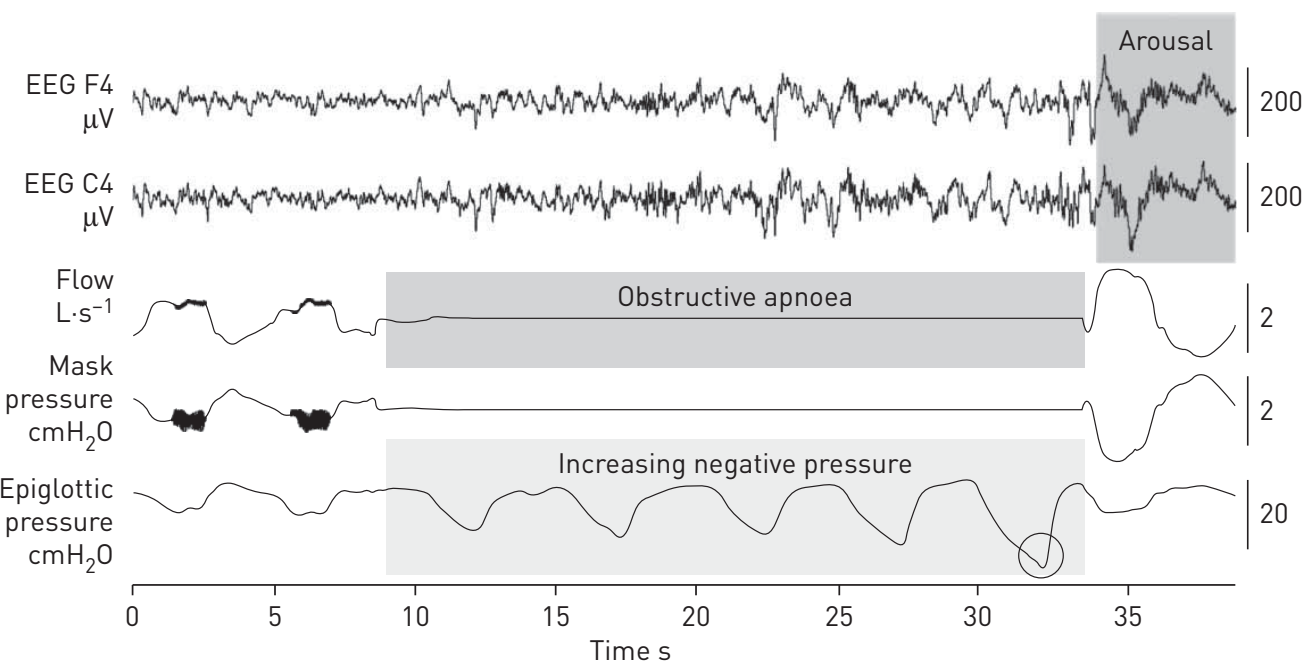

FIGURE 2 Polysomnographic recordings in an individual patient demonstrating how the respiratory arousal threshold measures were obtained. This example shows an $\sim 40$-s epoch of non-rapid eye movement N2 sleep in which the 35-year-old male patient (apnoea-hypopnoea index 20 events $\cdot \mathrm{h}^{-1}$; body mass index $32.2 \mathrm{~kg} \cdot \mathrm{m}^{-2}$ ) experienced an $\sim 25$-s respiratory event (shaded area of the respiratory flow channel labelled "Obstructive apnoea") that ended with a cortical arousal from sleep (shaded area of the two EEG channels labelled "Arousal"). The respiratory arousal threshold is quantified as the nadir epiglottic pressure during the respiratory effort occurring immediately prior to arousal (circled area on the epiglottic pressure channel, in this example approximately $-32 \mathrm{cmH}_{2} \mathrm{O}$ ). Flow is the nasal airflow measured via a mask and pneumotachograph; mask pressure is the pressure at the nasal mask measured using a pressure transducer. EEG: electroencephalogram. 
inability to sleep with the recording equipment during the physiology screening night (see figure 1 for further details). 31 individuals were randomised; 30 completed all 4 nights of the trial and were included in the final analyses (figure 1). Baseline anthropometric and sleep parameters were well matched between placebo and zopiclone groups (table 1). Anthropometric characteristics did not change during the 1-month trial (supplementary table E1). Adherence with the study intervention was high for both zopiclone and placebo groups $(93.7 \pm 9.7 \%$ and $93.4 \pm 11.0 \%$, respectively). Adverse events were uncommon during the 1-month trial, and all but one was minor and not related to the study medication (table 2). Table 3 summarises participant perceptions of the study medication (placebo or zopiclone) at the end of the 1-month trial. During zopiclone compared with placebo, $79 \%$ versus $56 \%$ reported improved sleep, $36 \%$ versus $31 \%$ said that they would continue with the study medication if available and $79 \%$ versus $69 \%$ correctly perceived which study medication they were taking.

\section{Respiratory events and sleep parameters}

The change in AHI from baseline to night 30 was not different between zopiclone and placebo conditions $(\mathrm{p}=0.24)$ (figure 3). The mean reduction in AHI at night 30 compared with baseline during zopiclone was $-5.9 \pm 10.2(\mathrm{p}=0.048)$ versus $-2.4 \pm 5.5$ events $\cdot \mathrm{h}^{-1}$ during placebo $(\mathrm{p}=0.098)$. Percentage reductions in AHI with zopiclone were similar in those with moderate $<-15$ and $\left.>-25 \mathrm{cmH}_{2} \mathrm{O}\right)$ and low $\left(\geqslant-15 \mathrm{cmH}_{2} \mathrm{O}\right)$ respiratory arousal thresholds (29\% versus $21 \%$ ) (table 4 ).

PSG data at baseline and night 30 during both conditions are summarised in table 4 . The increase in sleep efficiency from baseline to night 1 was significantly greater with zopiclone compared with placebo $(8.8 \pm 0.1 \%$ versus $1.2 \pm 0.1 \% ; \mathrm{p}=0.02)$ (supplementary table $\mathrm{E} 2)$. A comparable increase of $6.9 \pm 0.1 \%$ in sleep efficiency from baseline to night 30 with zopiclone occurred, but did not reach statistical significance $(p=0.06)$. The delta change from baseline to night 30 was not different between conditions (table 4). The amount of wake after sleep onset was reduced from baseline to night 1 with zopiclone versus placebo ( $-41 \pm 45$ versus $-8 \pm 38 \mathrm{~min}$; $\mathrm{p}=0.004$ ) (supplementary table E2), but not compared with night 30 (table 4 ). There were no between-condition differences in the majority of the other sleep parameters, including sleep onset latency, percentage total sleep time (TST) spent in non-rapid eye movement (REM) or REM sleep, arousal index, percentage TST supine, supine AHI, or respiratory event duration. Mean $\mathrm{SaO}_{2}$ during sleep at baseline was $\sim 1 \%$ lower in the zopiclone group ( $\mathrm{p}=0.03$ ). However, zopiclone did not alter mean or nadir $\mathrm{SaO}_{2}$. Sleep and respiratory parameters at night 1 were similar to night 30 (supplementary table E2).

\section{Sleepiness and alertness}

Sleepiness and alertness data from baseline to night 30 for placebo and zopiclone conditions are summarised in table 5. There were no differences in any measure of sleepiness or alertness, including ESS, FOSQ, Leeds, KSS or AusEd driving simulator performance, between conditions. Similarly, apart from an increase in the standard deviation from the median of the lane position during the driving task with zopiclone from baseline to night 1 compared with placebo $(6.7 \pm 8.4$ versus $-5.4 \pm 12.1 \mathrm{~cm}$; $\mathrm{p}=0.004)$ (supplementary table E3), measures of alertness and sleepiness on night 1 were not different between conditions.

\section{TABLE 1 Participant anthropometric and sleep characteristics}

\begin{tabular}{lcc} 
& Placebo & Zopiclone \\
\hline Participants & 16 & 14 \\
Sex & 13 & 12 \\
$\quad$ Male & 3 & 2 \\
$\quad$ Female & $46 \pm 13$ & $50 \pm 8$ \\
Age years & $29 \pm 4$ & $28 \pm 4$ \\
BMI $\mathbf{~ k g} \cdot \mathbf{m}^{-\mathbf{2}}$ & $21 \pm 10$ & $23 \pm 12$ \\
Mean AHI events $\mathbf{h}^{-1}$ & $6-38$ & $6-53$ \\
AHI range events $\cdot \mathbf{h}^{-1}$ & $-16 \pm 5$ & $-16 \pm 7$ \\
Arousal threshold $\mathbf{~} \mathbf{m}_{\mathbf{2}} \mathbf{0}$ & &
\end{tabular}

Data are presented as $\mathrm{n}$ or mean $\pm \mathrm{SD}$, unless otherwise stated. Group anthropometric and baseline obstructive sleep apnoea severity data acquired from the baseline and physiology (for respiratory arousal threshold data only) overnight sleep studies. BMI: body mass index; AHI: apnoea-hypopnoea index. 
TABLE 2 Adverse events reported during the 1-month trial

\begin{tabular}{|c|c|c|c|c|c|}
\hline & \multicolumn{2}{|c|}{ Placebo } & \multicolumn{2}{|c|}{ Zopiclone $^{\#}$} & \multirow[t]{2}{*}{ Related to zopiclone } \\
\hline & Minor & Serious & Minor & Serious & \\
\hline Abnormal dreams & $2 / 16$ & & $1 / 15$ & & Probably \\
\hline Abscess drainage & & & & $1 / 15$ & No \\
\hline Anxiety & & & $1 / 15$ & & Possibly \\
\hline Balance disorder & & & $1 / 15$ & & Probably \\
\hline Bradycardia & $1 / 16$ & & & & \\
\hline Change in sleep pattern & $3 / 16$ & & & & \\
\hline Constipation & $1 / 16$ & & & & \\
\hline Decreased appetite & & & $2 / 15$ & & Probably \\
\hline Diarrhoea & & & $1 / 15$ & & Probably \\
\hline Disturbance in memory or attention & & & $2 / 15$ & & Probably \\
\hline Dysgeusia & & & $3 / 15$ & & Definite \\
\hline Fatigue or asthenia & $1 / 16$ & & $1 / 15$ & & Probably \\
\hline Feeling abnormal & & & $2 / 15$ & & Probably \\
\hline Headache or migraine & & & $2 / 15$ & & Probably \\
\hline Insomnia & & & $2 / 15$ & & Possibly \\
\hline Joint swelling & & & $1 / 15$ & & No \\
\hline Loss of libido & & & $1 / 15$ & & Probably \\
\hline Muscle ache or injury & $1 / 16$ & & $1 / 15$ & & No \\
\hline Nasopharyngitis & $2 / 16$ & & $1 / 15$ & & No \\
\hline Oral ulcer & $1 / 16$ & & & & \\
\hline Rash & $2 / 16$ & & & & \\
\hline Stress & & & $1 / 15$ & & Possibly \\
\hline Weight loss & & & $2 / 15$ & & Possibly \\
\hline
\end{tabular}

Data represent adverse events reported during the 1-month trial or during the week after completion. None of these events required withdrawal from the study. A total of seven out of 16 participants randomised to placebo and 14 out of 15 randomised to zopiclone reported adverse events during the 1 -month trial. Six participants in the placebo group and eight in the zopiclone group reported more than one adverse event. ": one participant from the zopiclone group withdrew from the study prior to completing the 1-month trial due to nonmedical issues; ": whether each adverse event was related to zopiclone was determined by a physician according to the following criteria: not related ("No"), unlikely, possibly, probably or definitely.

Simple clinical tool to estimate the arousal threshold

Of the patients included in the 1-month trial, $100 \%$ were correctly identified as being eligible using the published algorithm [16] in combination with our inclusion criteria of an arousal threshold $\geqslant-25 \mathrm{cmH}_{2} \mathrm{O}$ and nadir $\mathrm{SaO}_{2} \geqslant 75 \%$. Applying these criteria for all screened participants, in whom data were available in $\mathrm{n}=60$, we found that only $15 \%$ would have been incorrectly enrolled. Of these, all had high arousal

TABLE 3 Participant feedback at study conclusion

Placebo

Zopiclone

\begin{tabular}{|c|c|c|}
\hline \multicolumn{3}{|c|}{ Did you feel like your sleep improved or worsened? } \\
\hline Improved & $9 / 16$ & $11 / 14$ \\
\hline Worsened & $1 / 16$ & $1 / 14$ \\
\hline Same & $6 / 16$ & $2 / 14$ \\
\hline \multicolumn{3}{|c|}{ If available, would you continue to use the study medication? } \\
\hline Yes & $5 / 16$ & $5 / 14$ \\
\hline No & $10 / 16$ & $9 / 14$ \\
\hline Unsure & $1 / 16$ & $0 / 14$ \\
\hline \multicolumn{3}{|c|}{ Do you feel like you were taking the sleeping pill or the placebo during the study? } \\
\hline Placebo & $11 / 16$ & $3 / 14$ \\
\hline Zopiclone & $5 / 16$ & $11 / 14$ \\
\hline
\end{tabular}

Data are presented as counts according to participant feedback over the 1-month trial. Data were collected on the morning of the final visit (night 30 ). 

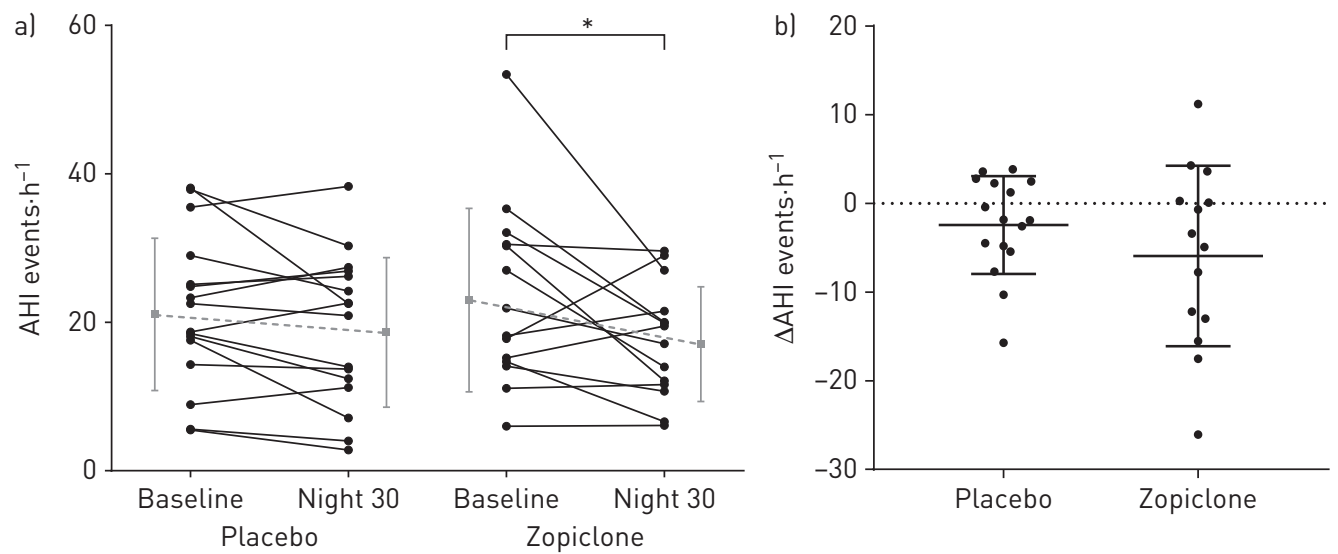

FIGURE 3 a) Apnoea-hypopnoea index (AHI) scatter plot representing each individual's AHI during placebo $(n=16)$ versus zopiclone $(n=14)$ conditions for baseline and night 30 . Grey lines and error bars indicate mean \pm SD. *: significant difference from baseline. b) Scatter plot indicating each individual's change in $\mathrm{AHI}(\Delta \mathrm{AHI})$ from baseline to night 30 for both conditions. Lines and error bars indicate mean \pm SD.

thresholds as measured with the epiglottic catheter $\left(-32 \pm 4 \mathrm{cmH}_{2} \mathrm{O}\right)$, which was underestimated by the algorithm $\left(-21 \pm 2 \mathrm{cmH}_{2} \mathrm{O}\right)$. These individuals also all had moderate to severe OSA (AHI $45 \pm 13$ events $\left.\cdot h^{-1}\right)$.

\section{Discussion}

The main findings of this study are that a 1-month course of a standard $7.5 \mathrm{mg}$ dose of nightly zopiclone does not worsen AHI, hypoxaemia, daytime sleepiness or morning alertness in individuals with OSA, lowmoderate respiratory arousal thresholds $\left(\geqslant-25 \mathrm{cmH}_{2} \mathrm{O}\right)$ and mild-moderate overnight hypoxaemia at baseline (nadir $\mathrm{SaO}_{2} \geqslant 75 \%$ ). In addition, there were no major adverse events with zopiclone use in this patient population. These novel findings extend recent single-night hypnotic studies and challenge earlier concerns that hypnotics systematically worsen OSA.

TABLE 4 Placebo and zopiclone polysomnography data for the 8-h overnight sleep studies during the baseline and night 30 conditions

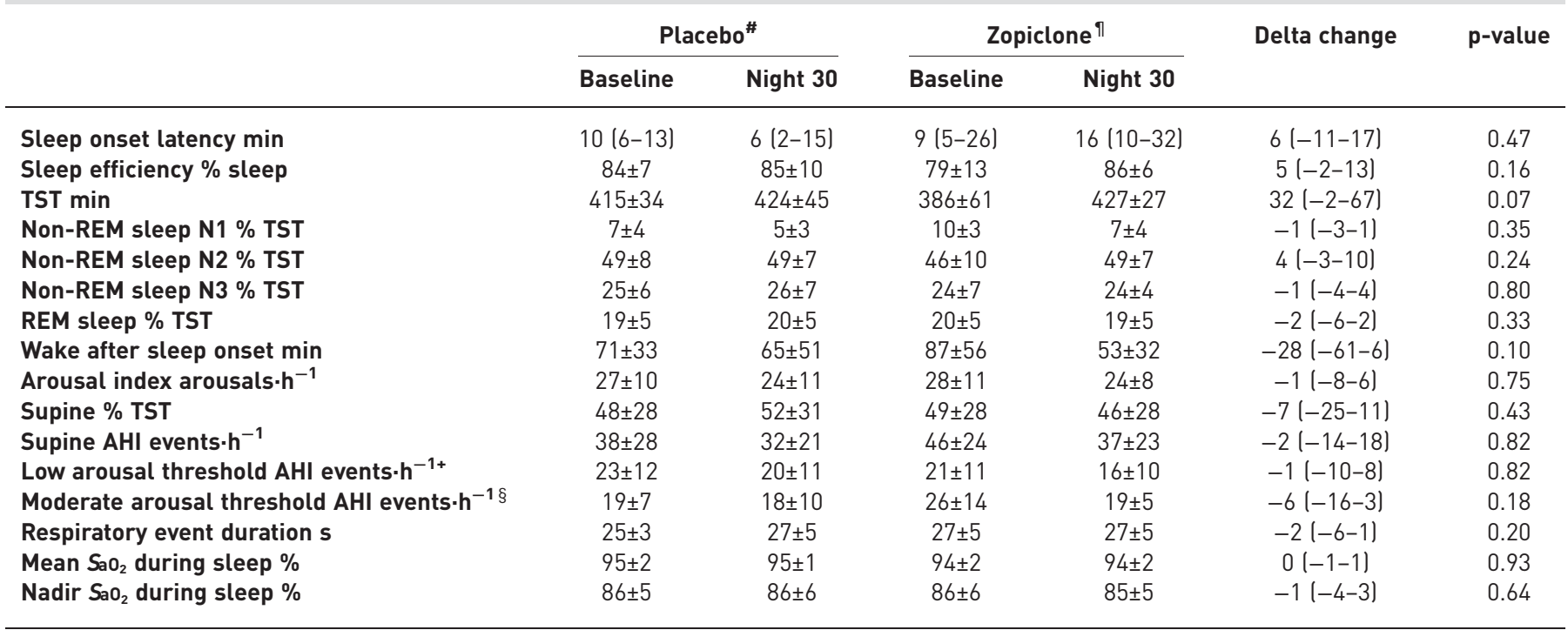

Data are presented as mean \pm SD or median (interquartile range), unless otherwise stated; the delta change column indicates the difference from baseline to night 30 between conditions (mean (95\% CI) for normally distributed data and Hodge-Lehmann estimated median $(95 \% \mathrm{CI}$ ) for nonnormally distributed data). REM: rapid eye movement; TST: total sleep time; AHI: apnoea-hypopnoea index; $\mathrm{SaO}_{2}$ : estimated arterial blood oxygen saturation (measured via pulse oximetry). ${ }^{\#}: \mathrm{n}=16 ;{ }^{9}: \mathrm{n}=14 ;{ }^{+}$: for low arousal threshold $\left(>-15 \mathrm{cmH}_{2} \mathrm{O}\right) \mathrm{AHI}, \mathrm{n}=9$ in the placebo group and $n=7$ in the zopiclone group; ${ }^{\S}$ : for moderate arousal threshold $<<-15$ and $\left.>-25 \mathrm{cmH}_{2} \mathrm{O}\right) \mathrm{AHI}, \mathrm{n}=7$ in the placebo and zopiclone groups. $p$-values refer to the difference from baseline to night 30 compared between placebo and zopiclone conditions. 
TABLE 5 Placebo and zopiclone sleepiness/alertness and driving simulator performance data collected during the baseline and night 30 conditions

\begin{tabular}{|c|c|c|c|c|c|c|}
\hline & \multicolumn{2}{|c|}{ Placebo ${ }^{\#}$} & \multicolumn{2}{|c|}{ Zopiclone $^{\pi}$} & \multirow[t]{2}{*}{ Delta change } & \multirow[t]{2}{*}{ p-value } \\
\hline & Baseline & Night 30 & Baseline & Night 30 & & \\
\hline ESS $^{+}$ & $5(4-12)$ & $6(4-11)$ & $8(5-12)$ & $7(4-9)$ & $-1(-4-1)$ & 0.55 \\
\hline $\mathrm{FOSQ}^{+}$ & $101 \pm 11$ & $101 \pm 12$ & $101 \pm 8$ & $103 \pm 11$ & $3(-2-7)$ & 0.25 \\
\hline Leeds $^{\S}$ & $5 \pm 1$ & $5 \pm 1$ & $4 \pm 1$ & $5 \pm 1$ & $0(-1-1)$ & 0.62 \\
\hline KSS & $6 \pm 2$ & $6 \pm 2$ & $6 \pm 1$ & $6 \pm 2$ & $0(-1-1)$ & $>0.99$ \\
\hline \multicolumn{7}{|l|}{ AusEd driving simulator } \\
\hline Deviation from median of lane $\mathrm{cm}$ & $59 \pm 22$ & $51 \pm 13$ & $48 \pm 16$ & $47 \pm 13$ & $7(-1-15)$ & 0.10 \\
\hline Deviation from $60-80 \mathrm{~km} \cdot \mathrm{h}^{-1} \mathrm{~km} \cdot \mathrm{h}^{-1}$ & $2 \pm 2$ & $2 \pm 1$ & $2 \pm 1$ & $2 \pm 1$ & $1(0-1)$ & 0.11 \\
\hline Braking reaction time $\mathrm{ms}^{+}$ & $1016 \pm 233$ & $1017 \pm 203$ & $1034 \pm 312$ & $957 \pm 206$ & $-77(-206-50)$ & 0.22 \\
\hline
\end{tabular}

Data are presented as mean \pm SD or median (interquartile range), unless otherwise stated; the delta change column indicates the difference from baseline to night 30 between conditions (mean $(95 \% \mathrm{CI}$ ) for normally distributed data and Hodge-Lehmann estimated median (95\% CI) for nonnormally distributed data). Epworth Sleepiness Scale (ESS) and Functional Outcomes of Sleep Questionnaire (FOSQ) data were collected in the evening $1.5 \mathrm{~h}$ prior to sleep; Leeds Sleep Evaluation Questionnaire (Leeds), Karolinska Sleepiness Scale (KSS) and AusEd data were collected 30 min after awakening. ${ }^{\#}: n=16$; ${ }^{\natural}: n=14 ;{ }^{+}$: for ESS, FOSQ and AusEd braking reaction time $n=15$ in the placebo group; ${ }^{\S}$ : for Leeds $n=13$ in the placebo group and $n=11$ in the zopiclone group. $p$-values refer to the difference from baseline to night 30 compared between placebo and zopiclone conditions.

\section{Effects of 1 month of nightly zopiclone on OSA severity}

Despite no systematic change versus placebo, the $25 \%$ within-group reduction in AHI over 1 month from baseline with zopiclone is consistent with recent single-night proof-of-concept clinical studies with eszopiclone and trazodone in predominantly unselected patients (23\% and 26\%, respectively) [14, 19]. However, this contrasts with no change in AHI in two small physiology studies with zopiclone versus placebo and trazodone versus baseline $[11,19]$. Unlike its stereoisomer eszopiclone, in which invariable reductions in AHI occurred in individuals with low arousal thresholds ( $45 \%$ overall) [14], this was not the case with zopiclone in the current study. Rather, similar reductions in AHI were observed in individuals with low and moderate arousal thresholds. The exact reasons for the apparent disparity between the studies are unknown. However, the causes of OSA vary between patients [12, 24]. Thus, factors beyond the arousal threshold that were not measured in the current study, such as between-patient differences in upper airway collapsibility and muscle responsiveness, may have contributed. Although eszopiclone is considered to be pharmacologically equivalent to zopiclone, subtle differences between the two agents may have also contributed [27]. However, standard doses of eszopiclone $(3 \mathrm{mg})$ and zopiclone increase the arousal threshold to a similar extent $(\sim 30 \%)[11,14,18]$. Finally, only one participant with a low arousal threshold had an increase in AHI with zopiclone. While supine sleep was not systematically different from baseline to night 30 for the group, this individual had a $>20 \%$ increase in supine sleep on night 30. Conversely, the participant with the greatest reduction in AHI with zopiclone had a $>20 \%$ decrease in supine sleep on night 30 . Thus, between-night changes in body position may be an important confounder at an individual level [28].

Another concern regarding hypnotic use in OSA is prolongation of respiratory event duration worsening blood gas disturbances. Indeed, previous studies indicate that certain hypnotics can worsen hypoxaemia, particularly at high doses or in obese individuals with very severe OSA $[9,11,21]$. Conversely, consistent with the current findings, several other hypnotic studies have shown no change in event duration or hypoxaemia in unselected patients and those selected on the basis of their baseline hypoxaemia [10, 11, $13,14,19,20,29-32]$.

\section{Sleep parameters}

Comparable to previous hypnotic studies $[10,11,14,30,32]$, a standard dose of zopiclone tended to improve sleep efficiency by $7-8 \%$ compared with placebo in the individuals with OSA we studied. This was also reflected in the subjective sleep quality reports at the study conclusion. Increased sleep efficiency combined with changes in OSA severity without causing blood gas disturbances may be beneficial for the substantial proportion $(\sim 40 \%)$ of OSA patients who also experience sleep disruption and symptoms of insomnia [6,7]. However, unlike the previous eszopiclone study where there was a significant shift from $\mathrm{N} 1$ to N2 sleep and reductions in the arousal index, which may have contributed to improvements in OSA severity [14], the proportion of time spent in non-REM and REM stages and the arousal index did not 
significantly change with zopiclone in the current study. This may be due to a ceiling effect, as sleep stage proportions were within or close to the normal range at baseline in the current study of predominantly mild-moderate severe patients versus the more severe patients in the eszopiclone study [14, 33]. Thus, inclusion of patients with poorer baseline sleep quality may yield significant improvements in sleep parameters with zopiclone. Alternatively, a standard dose of zopiclone may not be pharmacologically equivalent to previous eszopiclone studies in OSA $[14,21]$. Thus, while the potential for unwanted next-day side-effects may increase, a higher dose of zopiclone may be required to yield clinically relevant changes in sleep parameters, including AHI, in individuals with OSA.

\section{Sleepiness and alertness}

Hypnotics have generally been contraindicated in individuals with OSA due to concerns of harm relating to their central nervous system depressant properties, including the potential to exacerbate unwanted consequences such as next-day sleepiness and alertness. However, there are limited data on the effects of hypnotics on sleepiness and alertness in OSA. If hypnotics are to be considered as a therapeutic tool for certain individuals with OSA, it is crucial that they do not worsen next-day symptoms. The current study is the first to examine the effects of hypnotic use for an extended period on next-day sleepiness and alertness in individuals with OSA. Consistent with our previous single-night trial of a standard dose of zopiclone in unselected predominantly severe OSA patients, we found no change in next-day sleepiness as measured via the KSS [11]. Perceived sleepiness with the benzodiazepine nitrazepam [30] and next-day alertness and concentration with the melatonin receptor agonist ramelteon [34] also remained unchanged in acute single-night studies in individuals with OSA. In addition, none of the measures on the ESS, Leeds or FOSQ changed with zopiclone in the current study.

Furthermore, none of the objective measures of alertness assessed using the 30-min AusEd driving simulator performance test changed with zopiclone during the 1-month trial. This is crucial from a safety perspective as untreated OSA patients are already up to six times more likely to be involved in a traffic accident [3]. A lack of impairment in driving simulator performance in the current study is in contrast to previous studies in healthy participants in which standard doses of the " $\mathrm{z}$-drugs" zolpidem and zopiclone worsened on-road driving performance during an $\sim 60$-min task as well as next-day cognitive and psychomotor performance $[35,36]$. It has been proposed that healthy individuals may have exaggerated acute residual effects of hypnotics, which are not present in long-term hypnotic users or in individuals with poor sleep quality such as insomniacs and individuals with OSA, as potential negative residual effects may be outweighed by improvements in sleep quality [37]. Indeed, on-road driving performance during an $~ 60$-min driving task is not impaired in older insomniacs who use hypnotics frequently or infrequently after a standard dose of zopiclone [37]. Alternatively, as alertness declines with time during driving simulator tasks [38], it remains possible that driving impairment with zopiclone may occur beyond the 30 -min period tested in the current study. Future studies that incorporate other objective alertness assessments throughout the day (e.g. the maintenance of wakefulness test) would provide additional insight. It is also important to note that the majority of the individuals in the current study population were not particularly sleepy at baseline, and were asked to avoid confounding factors such as inadequate sleep opportunity and alcohol during the trial. Thus, next-day sleepiness following hypnotic use may differ in sleepy patients and with other confounders.

\section{Hypnotics and phenotyping}

Our secondary findings of a modest reduction in AHI from baseline in the current 1-month trial with zopiclone raise the possibility that the drug may be helpful in some individuals with OSA and insomnia. However, this remains to be tested in this population as symptoms of insomnia before versus after the trial were not systematically assessed. Furthermore, the reductions in AHI from baseline were of insufficient magnitude to be a clinically relevant therapeutic option as monotherapy to treat OSA for most patients. This is in contrast to a single-night standard dose of eszopiclone which invariably reduced AHI (by $\sim 45 \%$ overall) in patients with low arousal thresholds [14] and by $\sim 45 \%$ when combined with oxygen in unselected OSA patients [21]. Consistent with the importance of the underlying pathophysiological causes of OSA, responders to eszopiclone and oxygen (defined as patients with $>50 \%$ reduction in their AHI to $<15$ events. $h^{-1}$ ) had less collapsible upper airways, less severe OSA and greater upper airway muscle effectiveness [21]. These patients also tended to have low arousal thresholds [21]. Thus, these studies provide support for the potential use of hypnotics to reduce AHI to a therapeutic level when combined with other targeted supplementary therapies in patients who are carefully selected based on their underlying causes of OSA according to a phenotyping approach [23, 24]. However, further long-term studies with hypnotics that include additional phenotyping measures such as upper airway collapsibility are necessary to determine if AHI reductions are stable over time and apply to other clinically relevant targeted populations (e.g. sleepy patients and those aged $>65$ years in whom sleep disturbance is 
common). Thus, for hypnotics to be implemented safely and effectively clinically, simplified tools which allow for accurate identification of underlying pathophysiological causes of OSA are essential [39]. In accordance with this objective, the use of a recently developed tool to estimate the baseline arousal threshold using readily available variables from a diagnostic PSG was highly accurate when combined with nadir saturation criteria to identify suitable candidates for safe use of zopiclone in post hoc analyses in the current study. These findings highlight the potential to correctly estimate the arousal threshold in patients with less severe OSA in whom a hypnotic may be considered. However, patients with severe OSA with AHI $>30$ events $\cdot h^{-1}$ would benefit from a further PSG with a pressure catheter to accurately determine their arousal threshold prior to targeted treatment with hypnotics.

\section{Summary and clinical implications}

A standard dose of zopiclone taken nightly for 1 month does not worsen OSA severity, next-day sleepiness or alertness in selected OSA patients aged 18-65 years with low-moderate respiratory arousal thresholds without major hypoxaemia or sleepiness at baseline. In secondary analyses, there was also a tendency for zopiclone to reduce AHI by $\sim 25 \%$ and increase sleep efficiency from baseline. The findings of this randomised clinical trial challenge previous assumptions regarding the effects of hypnotics in individuals with OSA. Indeed, the American Academy of Sleep Medicine guidelines for the treatment of primary insomnia caution hypnotic use in patients with signs or symptoms of OSA [40]. The current findings suggest that screening to rule out severe overnight hypoxaemia combined with the use of a recently validated clinical tool from PSG data to estimate the arousal threshold may be helpful to accurately identify individuals in whom zopiclone can be administered safely for mild-moderate OSA. In particular, this may be relevant for the large number of OSA patients who also have insomnia symptoms, or fail to comply with continuous positive airway pressure or other therapies. Conversely, hypnotics should be avoided in OSA patients who have high arousal thresholds, long respiratory events and/or severe hypoxaemia at baseline, as these patients potentially may experience prolonged event durations and consequently more hypoxaemia with hypnotic use. The novel findings of the current study highlight the need for further research on targeted use of different hypnotics of varying doses and when combined with other therapies in individuals with OSA.

Acknowledgements: The authors thank the study pharmacist, Bandana Saini (University of Sydney, Sydney, Australia), for coordinating the randomisation process and blinding concealment. The authors would also like to thank Benjamin Tong (NeuRA, Randwick, Australia) and Anna Mullins (Woolcock Institute of Medical Research, Glebe, Australia) for scoring the overnight physiology and PSG studies, respectively.

Author contributions: S.G. Carter and D.J. Eckert wrote the manuscript, and all of the authors contributed to the final version and data interpretation. S.G. Carter, L.P. Fisher and C.M. Rollo collected and analysed the data. G. Cho, D.J. Stevens and A.L. D'Rozario assisted with data collection. D.J. Eckert and J.C. Carberry assisted with data collection and analysis, and in collaboration with R.R. Grunstein and D.K. McKenzie were responsible for the study conception and design.

Conflict of interest: S.G. Carter reports grants from the National Health and Medical Research Council (NHMRC; grant 1042493), and NeuroSleep, a NHMRC Centre for Research Excellence (grant 1060992), personal fees (Postgraduate Research Top-up Scholarship) from NeuroSleep, a NHMRC Centre for Research Excellence and personal fees (Supplementary Scholarship) from Neuroscience Research Australia (NeuRA), during the conduct of the study; and personal fees for part-time employment from ResSleep, outside the submitted work. J.C. Carberry reports grants from the NHMRC (grant 1042493) and NeuroSleep, a NHMRC Centre for Research Excellence (grant 1060992), during the conduct of the study. G. Cho reports grants from the NHMRC (grant 1042493) and NeuroSleep, a NHMRC Centre for Research Excellence (grant 1060992), during the conduct of the study. L.P. Fisher reports grants from the NHMRC (grant 1042493) and NeuroSleep, a NHMRC Centre for Research Excellence (grant 1060992), during the conduct of the study. C.M. Rollo reports grants from the NHMRC (grant 1042493) and NeuroSleep, a NHMRC Centre for Research Excellence (grant 1060992), during the conduct of the study. D.J. Stevens reports grants from the NHMRC (grant 1042493) and NeuroSleep, a NHMRC Centre for Research Excellence (grant 1060992), during the conduct of the study. R.R. Grunstein reports grants the NHMRC (grant 1042493) and NeuroSleep, a NHMRC Centre for Research Excellence (grant 1060992), during the conduct of the study, and personal fees for local advisory board activity from Merck and Teva, outside the submitted work. D.J. Eckert reports grants from the NHMRC (grant 1042493 and fellowship 1116942) and NeuroSleep, a NHMRC Centre for Research Excellence (grant 1060992), during the conduct of the study, and grants from Commonwealth Government of Australia Cooperative Research Centre Grant (industry partner Oventus Medical) and personal fees for advisory board activities and consultancy from Bayer, outside the submitted work. A.L. D'Rozario reports grants from the NHMRC (grant 1042493), and NeuroSleep, a NHMRC Centre for Research Excellence (grant 1060992), during the conduct of the study.

Support statement: This study was funded by a National Health and Medical Research Council (NHMRC) of Australia project grant (1042493) and NeuroSleep, a NHMRC Centre for Research Excellence (1060992). D.J. Eckert is supported by a NHMRC Senior Research Fellowship (1116942), R.R. Grunstein by a NHMRC Senior Principal Research Fellowship (1106974) and A.L. D’Rozario by a NHMRC-ARC Research Fellowship (1107716). Funding information for this article has been deposited with the Crossref Funder Registry. 


\section{References}

1 Vozoris NT, Leung RS. Sedative medication use: prevalence. risk factors, and associations with body mass index using population-level data. Sleep 2011; 34: 869-874.

2 Bertisch SM, Herzig SJ, Winkelman JW, et al. National use of prescription medications for insomnia: NHANES 1999-2010. Sleep 2014; 37: 343-349.

3 Teran-Santos J, Jimenez-Gomez A, Cordero-Guevara J. The association between sleep apnea and the risk of traffic accidents. Cooperative Group Burgos-Santander. N Engl J Med 1999; 340: 847-851.

4 Heinzer R, Vat S, Marques-Vidal P, et al. Prevalence of sleep-disordered breathing in the general population: the HypnoLaus study. Lancet Respir Med 2015; 3: 310-318.

5 McEvoy RD, Antic NA, Heeley E, et al. CPAP for prevention of cardiovascular events in obstructive sleep apnea. N Engl J Med 2016; 375: 919-931.

6 Beneto A, Gomez-Siurana E, Rubio-Sanchez P. Comorbidity between sleep apnea and insomnia. Sleep Med Rev 2009; 13: 287-293.

7 Krakow B, Melendrez D, Ferreira E, et al. Prevalence of insomnia symptoms in patients with sleep-disordered breathing. Chest 2001; 120: 1923-1929.

8 Guilleminault C. Benzodiazepines, breathing, and sleep. Am J Med 1990; 88: 25S-28S.

9 Berry RB, Kouchi K, Bower J, et al. Triazolam in patients with obstructive sleep apnea. Am J Respir Crit Care Med 1995; 151: 450-454.

10 Cirignotta F, Mondini S, Zucconi M, et al. Zolpidem - polysomnographic study of the effect of a new hypnotic drug in sleep apnea syndrome. Pharmacol Biochem Behav 1988; 29: 807-809.

11 Carter SG, Berger MS, Carberry JC, et al. Zopiclone increases the arousal threshold without impairing genioglossus activity in obstructive sleep apnea. Sleep 2016; 39: 757-766.

12 Eckert DJ, White DP, Jordan AS, et al. Defining phenotypic causes of obstructive sleep apnea. Identification of novel therapeutic targets. Am J Respir Crit Care Med 2013; 188: 996-1004.

13 Eckert DJ, Younes MK. Arousal from sleep: implications for obstructive sleep apnea pathogenesis and treatment. J Appl Physiol 2014; 116: 302-313.

14 Eckert DJ, Owens RL, Kehlmann GB, et al. Eszopiclone increases the respiratory arousal threshold and lowers the apnoea/hypopnoea index in obstructive sleep apnoea patients with a low arousal threshold. Clin Sci 2011; 120: 505-514.

15 Younes M. Role of arousals in the pathogenesis of obstructive sleep apnea. Am J Respir Crit Care Med 2004; 169: $623-633$.

16 Edwards BA, Eckert DJ, McSharry DG, et al. Clinical predictors of the respiratory arousal threshold in patients with obstructive sleep apnea. Am J Respir Crit Care Med 2014; 190: 1293-1300.

17 Gray EL, McKenzie DK, Eckert DJ. Obstructive sleep apnea without obesity is common and difficult to treat evidence for a distinct pathophysiological phenotype. J Clin Sleep Med 2017; 13: 81-88.

18 Carberry JC, Fisher LP, Grunstein RR, et al. Role of common hypnotics on the phenotypic causes of obstructive sleep apnoea: paradoxical effects of zolpidem. Eur Respir J 2017; 50: 1701344.

19 Eckert DJ, Malhotra A, Wellman A, et al. Trazodone increases the respiratory arousal threshold in patients with obstructive sleep apnea and a low arousal threshold. Sleep 2014; 37: 811-819.

20 Smales ET, Edwards BA, Deyoung PN, et al. Trazodone effects on obstructive sleep apnea and non-REM arousal threshold. Ann Am Thorac Soc 2015; 12: 758-764.

21 Edwards BA, Sands SA, Owens RL, et al. The combination of supplemental oxygen and a hypnotic markedly improves obstructive sleep apnea in patients with a mild to moderate upper airway collapsibility. Sleep 2016; 39 : 1973-1983.

22 Weaver TE, Grunstein RR. Adherence to continuous positive airway pressure therapy: the challenge to effective treatment. Proc Am Thorac Soc 2008; 5: 173-178.

23 Carberry JC, Amatoury J, Eckert DJ. Personalized management approach for obstructive sleep apnea. Chest 2018; 153: 744-755.

24 Eckert DJ. Phenotypic approaches to obstructive sleep apnoea - new pathways for targeted therapy. Sleep Med Rev 2018; 37: 45-59.

25 Vakulin A, Baulk SD, Catcheside PG, et al. Effects of alcohol and sleep restriction on simulated driving performance in untreated patients with obstructive sleep apnea. Ann Intern Med 2009; 151: 447-455.

26 Berry RB, Brooks R, Gamaldo CE, et al. The AASM Manual for the Scoring of Sleep and Associated Events: Rules, Terminology and Technical Specifications, Version 2.1. Darien, American Academy of Sleep Medicine, 2014.

27 Boyle J, Groeger JA, Paska W, et al. A method to assess the dissipation of the residual effects of hypnotics eszopiclone versus zopiclone. J Clin Psychopharmacol 2012; 32: 704-709.

28 Joosten SA, O’Driscoll DM, Berger PJ, et al. Supine position related obstructive sleep apnea in adults: pathogenesis and treatment. Sleep Med Rev 2014; 18: 7-17.

29 Jordan AS, O'Donoghue FJ, Cori JM, et al. Physiology of arousal in OSA and potential impacts for sedative treatment. Am J Respir Crit Care Med 2017; 196: 814-821.

30 Hoijer U, Hedner J, Ejnell H, et al. Nitrazepam in patients with sleep apnoea: a double-blind placebo-controlled study. Eur Respir J 1994; 7: 2011-2015.

31 Wang D, Marshall NS, Duffin J, et al. Phenotyping interindividual variability in obstructive sleep apnoea response to temazepam using ventilatory chemoreflexes during wakefulness. J Sleep Res 2011; 20: 526-532.

32 Rosenberg R, Roach JM, Scharf M, et al. A pilot study evaluating acute use of eszopiclone in patients with mild to moderate obstructive sleep apnea syndrome. Sleep Med 2007; 8: 464-470.

33 Berry RB. Fundamentals of Sleep Medicine. Philadelphia, Elsevier Health Sciences, 2011.

34 Kryger M, Wang-Weigand S, Roth T. Safety of ramelteon in individuals with mild to moderate obstructive sleep apnea. Sleep Breath 2007; 11: 159-164.

35 Mets MA, de Vries JM, de Senerpont Domis LM, et al. Next-day effects of ramelteon (8 mg), zopiclone (7.5 mg), and placebo on highway driving performance, memory functioning, psychomotor performance, and mood in healthy adult subjects. Sleep 2011; 34: 1327-1334. 
36 Leufkens TR, Lund JS, Vermeeren A. Highway driving performance and cognitive functioning the morning after bedtime and middle-of-the-night use of gaboxadol, zopiclone and zolpidem. J Sleep Res 2009; 18: 387-396.

37 Leufkens TR, Ramaekers JG, de Weerd AW, et al. On-the-road driving performance and driving-related skills in older untreated insomnia patients and chronic users of hypnotics. Psychopharmacology 2014; 231: 2851-2865.

38 Thiffault P, Bergeron J. Monotony of road environment and driver fatigue: a simulator study. Accid Anal Prev 2003; 35: 381-391.

39 Osman AM, Carter SG, Carberry JC, et al. Obstructive sleep apnea: current perspectives. Nat Sci Sleep 2018; 10 : 21-34.

40 Schutte-Rodin S, Broch L, Buysse D, et al. Clinical guideline for the evaluation and management of chronic insomnia in adults. J Clin Sleep Med 2008; 4: 487-504. 